\section{Ecthyma Gangrenosum in a Previously Healthy Infant}

Su Han Koo ${ }^{1}$, Joon Ho Lee ${ }^{1}$, Heakyeong Shin ${ }^{1}$, Jong Im Lee ${ }^{2}$

Departments of ${ }^{1}$ Plastic and Reconstructive Surgery, and ${ }^{2}$ Pathology, Dongguk University College of Medicine, Gyeongju, Korea

Correspondence: Heakyeong Shin

Department of Plastic and Reconstructive Surgery, Dongguk University College of Medicine, 87 Dongdae-ro, Gyeongju 780-350, Korea

Tel: +82-54-770-8242, Fax: +82-54-770-8501

E-mail: shinheakyeong@ hanmail.net

This article was presented as a poster at the Second Research and Reconstructive Forum of the Korean Society of Plastic and Reconstructive Surgeons on June 1-2, 2012 in Gwangju, Korea.

No potential conflict of interest relevant to this article was reported.

Received: 31 Aug 2012 • Revised: 26 Sep 2012 • Accepted: 26 Sep 2012 pISSN: 2234-6163• elSSN: 2234-6171

http://dx.doi.org/10.5999/aps.2012.39.6.673 • Arch Plast Surg 2012;39:673-675

Copyright (C) 2012 The Korean Society of Plastic and Reconstructive Surgeons This is an Open Access article distributed under the terms of the Creative Commons Attribution Non-Commercial License (http://creativecommons.org/licenses/by-nc/3.0/) which permits unrestricted non-commercial use, distribution, and reproduction in any medium, provided the original work is properly cited.
Ecthyma gangrenosum (EG) is a rare but characteristic cutaneous and potentially fatal disorder caused by a pseudomonal infection. The characteristic cutaneous lesion progresses sequentially, and starting as a maculopapular eruption, advancing to a hemorrhagic bulla, and then evolving into a gangrenous ulceration with black eschar surrounded by an erythema [1]. EG usually occurs in immunocompromised patients, but occasionally it affects previously healthy individuals. We present a case of a 10month-old previously healthy infant with transient severe neutropenia caused by influenza B infection without bacteremia.

A previously healthy 10-month-old boy was admitted with a 5-day history of fever, general weakness, and a red spot on the right buttock. The red spot had started as a small papule, after a perineal diaper rash disappeared. Initially, it was thought to be part of the diaper rash, but rapidly progressed to a bulla and finally to a necrotic black eschar with surrounding erythema within only 24 hours, but without suppuration (Fig. 1).

An initial blood test revealed leukopenia (4,000/ $\mu \mathrm{L}$; normal range, 6,000 to $17,500 / \mu \mathrm{L}$ ) with severe neutropenia (absolute neutrophil count, $320 / \mu \mathrm{L}$; normal range, 3,150 to $6,200 / \mu \mathrm{L}$ ), lymphocytosis, and monocytosis. His inflammatory markers were elevated: C-reactive protein $13.9 \mathrm{mg} / \mathrm{dL}$ (normal range, 0.08 to $1.12 \mathrm{mg} / \mathrm{dL}$ ) and erythrocyte sedimentation rate $35 \mathrm{~mm} / \mathrm{hr}$ (normal range, 0 to $9 \mathrm{~mm} / \mathrm{hr}$ ). All other results were in the normal range. However, a nasal smear test revealed positivity for influenza B.

At the time of admission, the child was initially given intravenous third-generation cephalosporin for broad spectrum coverage, but the cutaneous lesion continued to progress. A wound culture grew Pseudo-

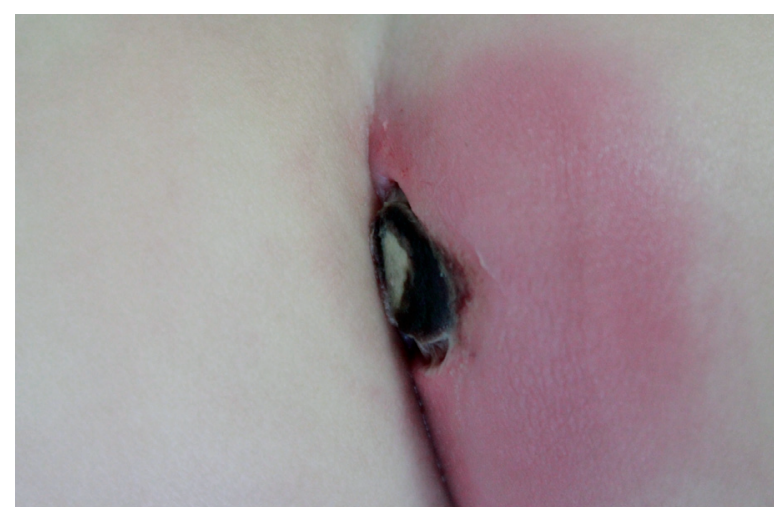

Fig. 1.

Cutaneous lesion. Typical appearance of ecthyma gangrenosum on the right buttock. 
monas aeruginosa 2 days later, but repeated blood cultures grew nothing. However, a skin biopsy showed a necrotizing small vessel with thrombus surrounded by bluish bacterial clumps (Fig. 2). In light of the wound culture result, intravenous antibiotics were changed to piperacillin plus tazobactam, and ceftazidime was started additively.

After changing antibiotics, the buttock lesion rapidly improved. Initially the erythema around the eschar faded and the central necrosis stopped enlarging, and remained at a size of about $2.5 \mathrm{~cm}$ in diameter (Fig. 3). Surgical repair was planned when the lesion became stable. A local flap using an intergluteal fold line was performed for wound coverage (Fig. 4). The flap survived without complication and the wound healed well.

In general, EG is pathognomonic for Pseudomonas septicemia, but rarely, it occurs by direct skin inoculation without bacteremia [1]. In our case, a single lesion, a previous history of diaper rash and the absence of bacteremia suggest the latter etiology.

Lesions progress in a characteristic sequential manner. They start as papules and advance to hemorrhagic bullae, and then evolve into gangrenous ulcerations with black eschar surrounded by an erythematous halo [1]. EG can be distinguished from pyoderma gangrenosum by a greater number of suppurative lesions and no bacterial pathogen in cultures, and can also be distinguished from common cellulitis by the characteristic sequential pattern and the speed of disease progression, which might be a key to its early recognition and treatment. Lesions can occur anywhere, but are more common in the perineum, buttocks, axillae, and extremities [2].

The histopathologic appearance of EG is a vasculitis caused by bacterial invasion of themedia and adventitia of the vascular wall, sparing the intima. The intraluminal thrombus causing locoregional necrosis is caused by bacilli in the vessel walls, by circulating immune complexes, and/or by the effect of bacterial exotoxins or endotoxins [2]. This feature explains the rapid progression of the lesions to necrosis. In our case, a necrotizing small vessel with intraluminal

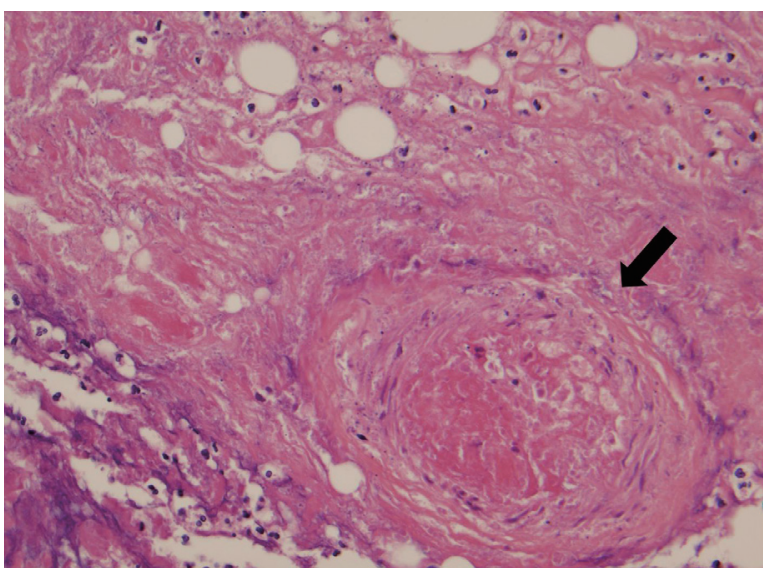

Fig. 2.

Histological finding. A necrotizing small vessel with thrombus surrounded by bluish bacterial clumps $\left(H \& E_{1} \times 400\right)$.

Fig. 4.

Postoperative view. (A) Immediate postoperative view, and (B) View at 1 month postoperatively.

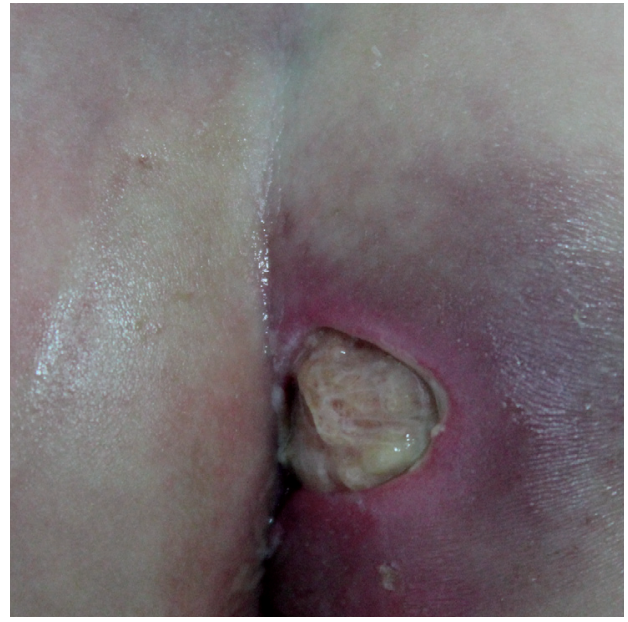

Fig. 3. After changing to anti-pseudomonal antibiotics. The improvement in surrounding erythema. 
thrombus surrounded by bluish bacterial clumps was observed (Fig. 2).

Although EG occurs in $1 \%$ to $30 \%$ of cases of Pseudomonas sepsis as a first manifestation of immunodeficiency or in patients with chronic disease, cases have occasionally been reported in previously healthy individuals. These individuals had undetected immunodeficiency or were transiently immunocompromised due to drugs, infections, or some other cause. Thus, an immunological work up should be performed in suspected cases [3]. In our patient, influenza B infection caused severe neutropenia and a previous diaper rash might have aided bacterial inoculation. A subsequent immunological work up established that the infant had a normal immune system.

In cases of EG, early recognition and the administration of prompt appropriate antibiotics is essential due to rapid disease progression. Antibiotics including ceftazidime (alone), azlocillin, mezlocillin or piperacillin-tazobactam with aminoglycosides are known to be effective [4]. Furthermore, in several of the reported cases, after controlling the infection, wounds healed by secondary intention left notable scars. Mortality rates of from $38 \%$ to $96 \%$ have been reported in septicemic cases and of $15 \%$ in nonbacteremic cases, respectively [5]. Poor prognosis is associated with multiple lesions, delay in treatment, and neutropenia.

In conclusion, this case shows that even a previously healthy patient in a temporarily immunocompromised state may be susceptible to pseudomonal infections, which cautions that the possibility of opportunistic infections warrants consideration. The characteristic clinical features of the skin lesion led to a differential diagnosis of EG. Furthermore, the rapid progression of the lesions and EG's high mortality rate emphasize the importance of early suspicion and proper treatment even when diagnosis has not been confirmed.

\section{References}

\section{Boisseau AM, Sarlangue J, Perel Y, et al. Perineal} ecthyma gangrenosum in infancy and early childhood: septicemic and nonsepticemic forms. J Am Acad Dermatol 1992;27:415-8.

2. Mota-Burgos A, Villa AV, Noguera-Julian A, et al. Fever and skin lesions in a healthy 6-month-old boy. Diagnosis: Ecthyma gangrenosum. Pediatr Infect Dis J 2012;31:789-94.

3. Zomorrodi A, Wald ER. Ecthyma gangrenosum: considerations in a previously healthy child. Pediatr

Infect Dis J 2002;21:1161-4.

4. Kliegman RM, Behrman RE, Jenson HB, et al. Nelson textbook of pediatrics. 18th ed. Philadelphia: Elsevier Saunders; 2007.

5. Huminer D, Siegman-Igra Y, Morduchowicz G, et al. Ecthyma gangrenosum without bacteremia. Report of six cases and review of the literature. Arch Intern Med 1987;147:299-301. 